\title{
Índice espectral de vegetación para la caracterización simultánea de la floración y crecimiento vegetal
}

\author{
Development of a spectral vegetation index for simultaneous characterization \\ of flowering and foliage development
}

\author{
Marcos Casiano ${ }^{1}$ y Fernando Paz $^{2 *}$
}

1 Programa Mexicano del Carbono. Chiconautla No. 8. Col. Lomas de Cristo. 56230 Texcoco, Edo. de México, México.
${ }^{2}$ GRENASER, Colegio de Postgraduados. Carretera México-Texcoco km 36.5. 56230 Montecillo, Texcoco, Edo. de México, México.
${ }^{\ddagger}$ Autor responsable (ferpazpel@gmail.com)

\begin{abstract}
RESUMEN
Es importante caracterizar los patrones fenológicos de la floración y desarrollo del follaje en la vegetación con algún grado de caducidad, debido a su relación con el ciclo del carbono y del agua. Las técnicas de campo y el uso de fotografías digitales en torres de observación tienen alcances geográficos limitados, por lo que la tecnología de los sensores remotos es una opción costo-efectiva. En este trabajo se revisó un modelo fenológico floración-follaje de desarrollo previo y se presenta un nuevo modelo que incluye el uso de un índice de vegetación de floración y follaje (IVFF) para caracterizar y diferenciar los patrones fenológicos. Para correlacionar el IVFF con la cobertura de la floración (CF) en la vegetación, se desarrolló un experimento de validación que incluyó un arbusto de bajo porte, en el cual se colocaron diferentes coberturas $(<15 \%)$ de flores blancas. Los resultados mostraron que el IVFF se correlaciona linealmente con la cobertura de flores, y que la relación depende de la cobertura del follaje donde se colocan. El desarrollo propuesto permitió establecer correlaciones claras entre el IVFF y la cobertura floral de la vegetación con diferentes coberturas aéreas, por lo que fue significativamente eficaz, aún con porcentajes bajos de floración, imposibles de detectar con otras técnicas. En las estimaciones sólo se utilizan las bandas del rojo y el infrarrojo cercano, por lo que convierte al método propuesto en una herramienta asequible para la mayoría de las aplicaciones de sensores remotos.
\end{abstract}

Cita recomendada:

Casiano, M. y F. Paz. 2018. Índice espectral de vegetación para la caracterización simultánea de la floración y crecimiento vegetal. Terra Latinoamericana 36: 169-181.

DOI: https://doi.org/10.28940/terra.v36i2.249
Palabras clave: sensores remotos, IVFF, patrón artificial de floración, IVISn, reflectancias normalizadas y estandarizadas.

\section{SUMMARY}

It is important to characterize the phenological patterns of flowering and foliage development in vegetation that has some degree of deciduous behavior due to its relation to the carbon cycle and water. Field techniques and use of digital photograph observation towers have limited geographical scope, so that remote sensing technology is a cost-effective option. In this work a flowering-foliage phenology model was revised and a new model that includes the use of vegetation index of flowering and foliage (VIFF) to characterize and differentiate the patterns is presented. To correlate the VIFF coverage flowering (CF) in vegetation, we conducted a validation experiment that included a lowgrowing shrub, in which different coverages $(<15 \%)$ of white flowers were placed. The results showed that the VIFF correlates linearly with the coverage of flowers and the relationship depends on the coverage of the foliage where they are placed. The proposed development allowed establishing clear correlations between VIFF and floral vegetation cover of different coverage area, which was significantly effective, even with low percentages of flowering, undetectable with other techniques. Because it uses only red and near infrared bands, the proposed tool is accessible for most applications of remote sensing.

Recibido: abril de 2017. Aceptado: marzo de 2018. Publicado en Terra Latinoamericana 36: 169-181. 
Index words: remote sensing, IVFF, artificial flowering pattern, IVISn, normalized and standardized reflectances.

\section{INTRODUCCIÓN}

La caracterización de los patrones fenológicos de la vegetación permite estimar su impacto en la viabilidad reproductiva y adaptativa, así como en los balances de agua y energía (Running y Nemani, 1991; White et al., 1999). El cambio climático puede alterar la fenología de la vegetación, lo que conlleva cambios en la distribución espacial, tiempo de presentación $\mathrm{y}$, duración de los diferentes estadios fenológicos (Peñuelas y Filella, 2001; Schwartz et al., 2006; Cleland et al., 2007). Particularmente, los bosques tropicales con algún nivel de caducidad del follaje, son de alto interés por su variabilidad fenológica ante factores internos y externos (Sakai, 2001).

Los patrones fenológicos de la vegetación se pueden caracterizar en función del tiempo de presentación, duración, frecuencia, amplitud, sincronía y regularidad de los estadios (Gentry, 1974; Opler et al., 1980; Newstrom et al., 1994), procesos que generalmente se correlacionan entre sí (Bawa et al., 2003) y que dan como resultado una amplia diversidad de patrones en bosques tropicales (Sakai, 2001), y otros tipos de vegetación. Numerosos autores atribuyen a diversos factores bióticos y abióticos, la variedad de patrones fenológicos de desarrollo del follaje y floración (Kochner y Handel, 1986; Bawa, 1990; van Schaik et al., 1993; Wright y van Schaik, 1994; Sakai, 2001; Boyle y Bronstein, 2012), pero en muchos casos lo que se observa no concuerda con las predicciones.

La caracterización de la fenología de la vegetación se puede hacer directamente en campo (Borchert, 1983; Heideman, 1989; Bullock y Solis-Magallanes, 1990), lo que implica altos costos de implementación y áreas de observación reducidas. Una alternativa para contrarrestar estas limitaciones es tomar fotografías digitales con cámaras que se instalan en estructuras por encima de la vegetación (Richardson et al., 2009; Parihar et al., 2013) y caracterizar la fenología a partir de las imágenes, pero la técnica aún conserva el problema de la limitación geográfica. Otra opción es la tecnología de los sensores remotos que, dado su enfoque espacial exhaustivo y la temporalidad de las visitas a un mismo pixel, plantea una solución eficiente y de bajo costo (Zhang et al., 2012).
En relación al uso de sensores remotos para la modelación de la fenología se utilizan diferentes índices de vegetación o IV (Zhang et al., 2012), de los que sobresalen el NDVI Rouse et al. (1974), el EVI (Liu y Huete, 1995) y el EVI2 (Jiang et al., 2008), en los que los datos se filtran para reducir su variabilidad (Kathuroju et al., 2007; Geerken, 2009; Atkinson et al., 2012) y poder detectar umbrales que definan los estadios fenológicos (Zhang et al., 2003 y 2012). Con relación a la fenología del follaje, los IV permiten definir los patrones fenológicos de ecosistemas o biomas a escala regional o global (Zhang et al., 2004; Stöckli y Vidale, 2004; Zhang et al., 2006). El crecimiento y senescencia del follaje se analiza por los IV que se basan en el alto contraste entre las bandas espectrales del rojo (R) e infrarrojo cercano (IRC), para diferenciar la vegetación de otros objetos terrestres (Tucker, 1979). En el caso de la floración, las bandas que mejor caracterizan la fenología también se asocian al R e IRC (Wouters et al., 2013), aunque en fotografías digitales (solo bandas del visible) se usan IV sin el IRC (Richardson et al., 2009; Parihar et al., 2013) y, aún en el caso de contar con las bandas del IRC, los índices solo usan las bandas del visible (Ge et al., 2006; Chen et al., 2009), lo que refleja la idea de que la floración puede distinguirse en función de los colores de las flores. En el caso de sensores remotos, las bandas del visible (rojo, verde y azul) tienen efectos atmosféricos importantes provenientes de aerosoles (Kaufman y Tanre, 1996), lo que limita su uso en la detección de la floración.

Con el objetivo de desarrollar un índice de la vegetación que detecte la floración y la diferencie del follaje, en este trabajo se presenta el desarrollo de las bases teóricas de un IV orientado a tal objetivo y se analiza su viabilidad utilizando mediciones de un experimento con una especie arbustiva donde se adicionaron diferentes cubrimientos de flores, dispuestas en un arreglo aleatorio y con agrupación de flores. En términos genéricos, el experimento puede interpretarse como mezclas de follaje y flores, representando diferentes tipos de vegetación, incluidos los bosques tropicales caducifolios, donde se busca detectar la presencia de flores con bajo cubrimiento del follaje. 


\section{FUNDAMENTO TEÓRICO}

\section{Patrones Fenológicos y su Caracterización con Índices Espectrales de Vegetación}

Si se considera la evolución temporal de la vegetación, las condiciones de iluminación solar cambian, lo que introduce efectos que distorsionan los valores del R e IRC. Para tomar en cuenta este efecto, conviene estandarizar las condiciones, lo que suele hacerse con un modelo simplificado de geometría de iluminación-visión (Bolaños y Paz, 2010):

$$
\begin{aligned}
& x=90-\theta v+\theta s \\
& R n=\ln (R) \cos (x) \\
& x=90-g R n
\end{aligned}
$$

donde: $\theta \mathrm{v}$ es el ángulo cenital de visión, $\theta \mathrm{s}$ el de iluminación solar, $\chi$ es una variable angular de posición, $R$ es la reflectancia de cualquier banda, $R n$ es la reflectancia normalizada por posición angular y efecto de escala (función logaritmo natural) y $g$ es un parámetro que define la geometría sol-sensor. La ventaja del modelo es que solo tiene un parámetro, el cual puede estimarse con un solo dato de medición. En lo siguiente solo se considerarán reflectancias normalizadas y estandarizadas a la geometría $\theta \mathrm{v}=0^{\circ} \mathrm{y} \theta \mathrm{s}$ $=30^{\circ}$, de tal forma que todas las mediciones espectrales tienen una base común de observación. La inversión del modelo de geometría sol-sensor, relaciones [1], para estimar reflectancias no normalizadas, introduce errores (Bolaños y Paz, 2010), y su uso en índices de vegetación no es adecuada. De esta forma, el índice de vegetación IVIS (Paz et al., 2011) se define en términos de reflectancias normalizadas y estandarizadas:

$$
\begin{aligned}
& I V I S n=-\ln \left(\frac{d I R C n \infty-d I R C n}{d I R C n \infty-d I R C n s}\right) \\
& d I R C n=I R C n-\left(a_{n S}+b_{n S} R n\right)
\end{aligned}
$$

Los parámetros se definieron como $d I R C n s=0$, $d I R C n \infty=3, a_{\mathrm{nS}}=0$ y $b_{\mathrm{nS}}=0.8$ y se emplean en la siguiente discusión.

\section{Follaje de la Vegetación}

La Figura 1 muestra, en forma esquemática, la evolución temporal de un índice de vegetación asociado al crecimiento del follaje y su senescencia, en vegetación perenne (Figura 1a) y caducifola (Figura 1b). El patrón que se observa es característico cuando se usan sensores remotos (Zhang et al., 2004; Zhang et al., 2006), aun cuando generalmente emerge en forma definida después de remover los ruidos espectrales y suavizar los datos (Kathuroju et al., 2007; Geerken, 2009; Atkinson et al., 2012).

En el caso de vegetación con diferentes niveles de caducidad del follaje o mezcla de perenne y caducifolia, el patrón temporal resulta intermedio (Zhang et al., 2006), lo que se reconoce por la diferencia de los días julianos entre el IV máximo y el mínimo (Cuba et al., 2013). Los patrones de la Figura 1b permiten definir los umbrales y su duración en los diferentes estadios del desarrollo del follaje, en función de la evolución temporal del IV (Zhang et al., 2012).

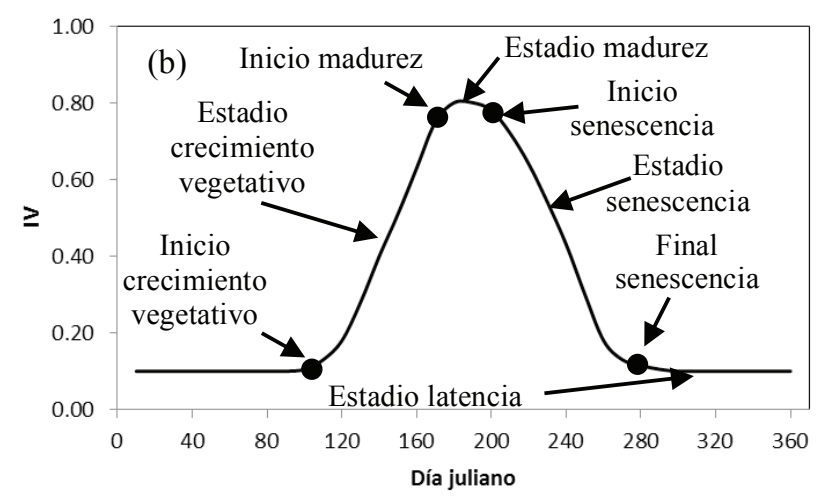

Figura 1. Patrones esquemáticos de la evolución temporal de un índice de vegetación (IV) en vegetación perenne (a) y caducifolia (b). La figura 1b, muestrea el inicio del crecimiento vegetativo [1], Estadio de crecimiento vegetativo [2], Inicio de la madurez [3], Estadio máximo de madurez [4], Inicio de senescencia [5], Estadio de senescencia [6], Final de senescencia [7] y Estado de latencia [8]. 
El patrón de la Figura 1b es propio del crecimiento y senescencia del follaje de los diferentes tipos de vegetación. Por ejemplo, la Figura 2 muestra la evolución temporal de Rn e IRCn, así como del IVISn, para el caso de un cultivo de frijol, cuyo experimento está descrito en Reyes et al. (2011).

En la Figura 2 las reflectancias $\mathrm{Rn}$ e IRCn presentan patrones contrarios en la etapa vegetativa $\mathrm{y}$ de senescencia, por lo que el IVISn exhibe un patrón de crecimiento y decrecimiento similar al de la Figura $1 \mathrm{~b}$. El patrón de la Figura 2, de evolución temporal de reflectancias normalizadas o no, es similar al que se observa para el caso de bosques (Nilson et al., 2012) y vegetación herbácea (Hunt y Williams, 2006; Chen et al., 2009).

\section{Floración de la Vegetación}

Con información de experimentos provenientes de diferentes componentes de la vegetación (material muerto, hojas, yemas y flores) en arreglos que aproximan las etapas fenológicas de la floración, Casiano y Paz (2014) plantearon un modelo en el que se usa el índice IVISn, (Figura 3).

En la Figura 3, el inicio (inducción) de la floración lo constituye el crecimiento de brotes foliares y material fotosintético, lo que corresponde con la parte de crecimiento del IVISn hasta un valor máximo; después se produce un decrecimiento del IVISn hasta un valor mínimo, donde la floración finaliza y, de ahí en adelante, el índice refleja el crecimiento del follaje. El patrón de la floración que se muestra en la Figura 3 solo se detecta cuando la floración es intensa y sincrónica, además de que el fondo de la vegetación debe ser obscuro ya que las tonalidades claras enmascaran el color de las flores y sus mezclas.

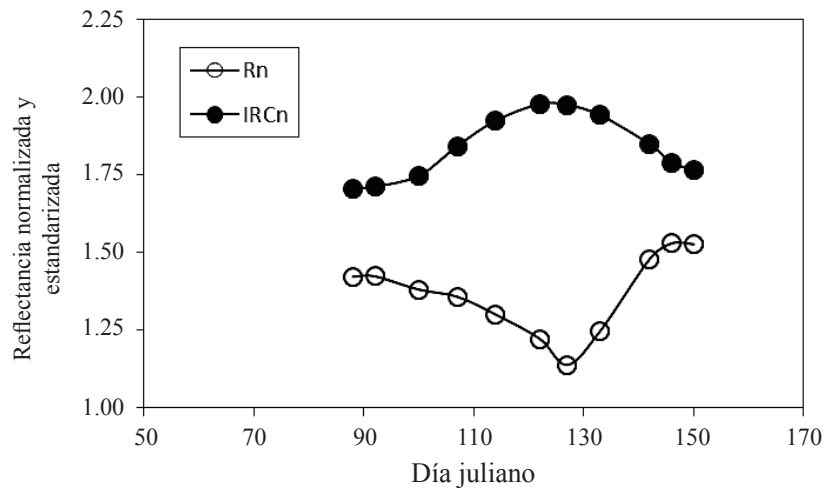

La Figura 4 muestra los patrones del Rn, IRCn e IVISn del periodo de floración del experimento de Casiano y Paz (2014) para los estadios fenológicos 1 al 5 ( 1 = inicio, 3 = pico, 5 = final $)$, con flores blancas y amarillas. El uso de flores lilas mostró un patrón similar al observado en la Figura 4.

De acuerdo con la Figura 4, los patrones del Rn e IRCn crecen y decrecen simultáneamente con la fenología (y el tiempo) de la floración, inverso a lo que sucede con el follaje (Figura 2). Este patrón es similar al que se observó en experimentos de floración (número de flores) en vegetación herbácea, en donde con el incremento de la floración, las bandas del $\mathrm{R}$ e IRC crecen simultáneamente (Hunt y Williams, 2006; Chen et al., 2009). En bosques, Nilson et al. (2012) mostraron que la banda del $\mathrm{R}$ se incrementa y disminuye durante la ocurrencia de la floración, en forma similar a lo que sucede con la floración en vegetación herbácea, pero la banda del IRC, con ruido, muestra un patrón no sincronizado en tiempo, aparentemente, con la banda del R. Algo similar se observó en vegetación herbácea (Hunt y Williams, 2006).

Es posible distinguir en la Figura 4, que el IVISn decrece hasta el pico de la floración y después crece hasta el término de la misma; aunque para el caso de flores de color amarillo, este comportamiento está ausente y es contrario a lo esperado, lo que puede atribuirse a que este color tiene un patrón de reflectancia similar al suelo y material muerto, donde la señal de las hojas coexistentes con el pico de floración es dominante.

Aún cuando el modelo propuesto para detectar la floración con el IVISn, (Figura 3), es una aproximación indirecta razonable, es importante desarrollar un índice espectral que capture los patrones fenológicos de la floración y del follaje, capaz de distinguirlos entre sí.

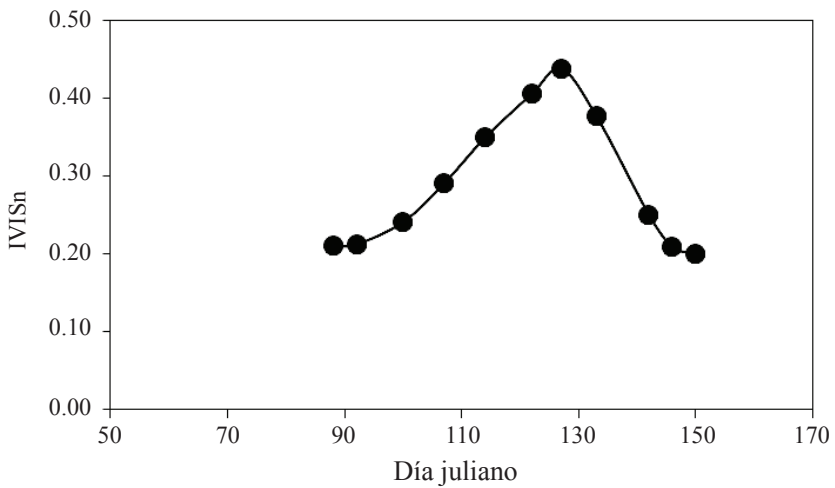

Figura 2. Evolución temporal de Rn, IRCn e IVISn para el caso de un cultivo de frijol (adaptado de Reyes et al., 2011). 


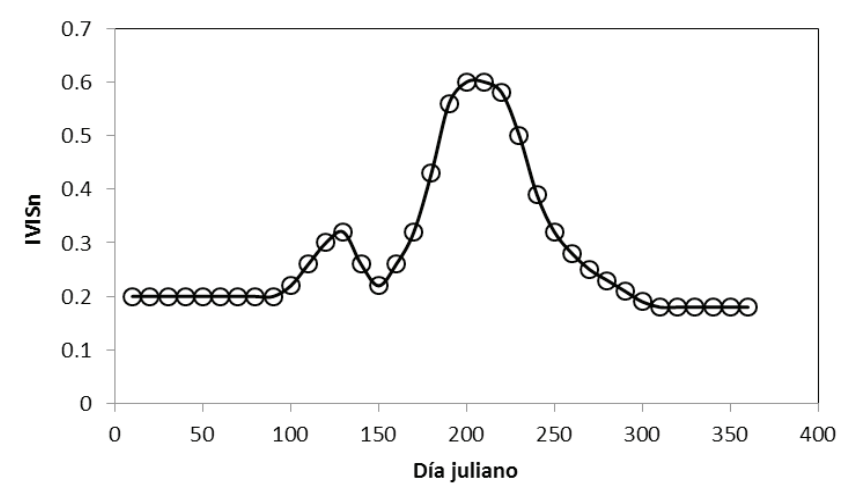

Figura 3. Esquematización de los estadios de la floración en vegetación caducifolia (Casiano y Paz, 2014).

\section{Desarrollo de un Índice Espectral para Floración y Follaje}

Para desarrollar un índice espectral de floración y follaje (IVFF) se requiere caracterizar los patrones del Rn e IRCn de sus respectivas fenologías. En el caso de la floración, ambas bandas crecen y decrecen simultáneamente, por lo que el término (Rn)(IRCn) captura este patrón. Así, para valores pequeños del término, se puede representar el inicio y final de la floración y, para valores grandes, los casos de mayor floración. Para el follaje, la banda del IRCn crece y la del $\mathrm{Rn}$ decrece en la etapa vegetativa y el patrón se revierte en la de senescencia. El término IRCn - Rn captura el patrón, en donde los valores pequeños representan el inicio y fin del crecimiento del follaje $\mathrm{y}$, los valores altos, follaje mayor. Para representar ambos patrones se desarrolló el índice de vegetación de floración y follaje:

$$
I V F F=\frac{(R n)(I R C n)}{I R C n-R n}
$$

La ventaja del IVFF en el formato de razón es que, cuando se presenta el patrón de floración, se tiene un crecimiento y decrecimiento del índice, (Figura 5); contrario al follaje, (Figura 6), en donde la etapa vegetativa tiene un patrón decreciente y la de senescencia uno creciente, lo que distingue claramente el dominio de los patrones (hojas o flores).

El modelo de floración y follaje propuesto se muestra en la Figura 7.

Si se cambia el numerador por el denominador en la definición del IVFF en la ecuación [3], se obtienen patrones contrarios (Figura 7).
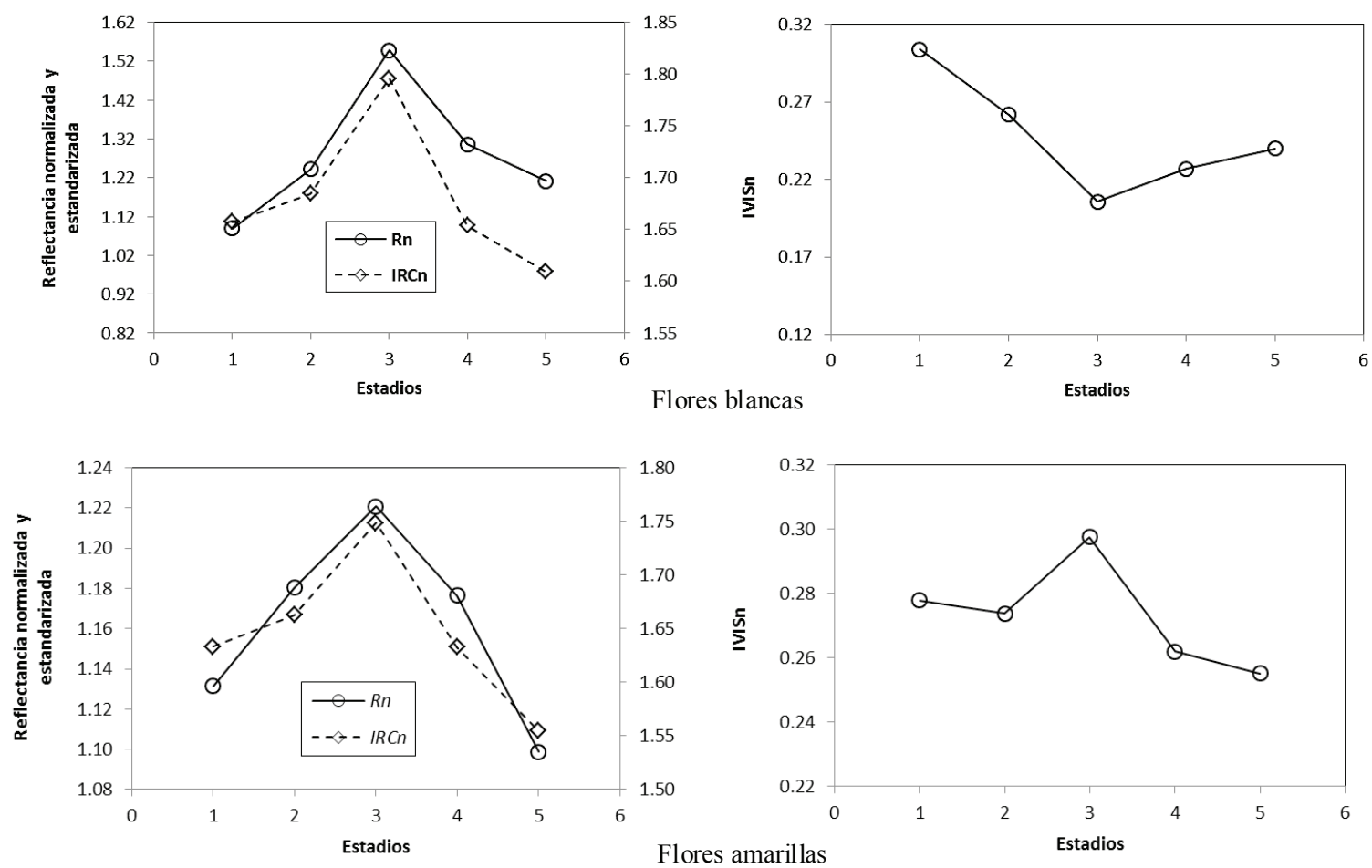

Figura 4. Patrones fenológicos del Rn, IRCn e IVISn para flores blancas y amarillas (adaptado de Casiano y Paz, 2014). 

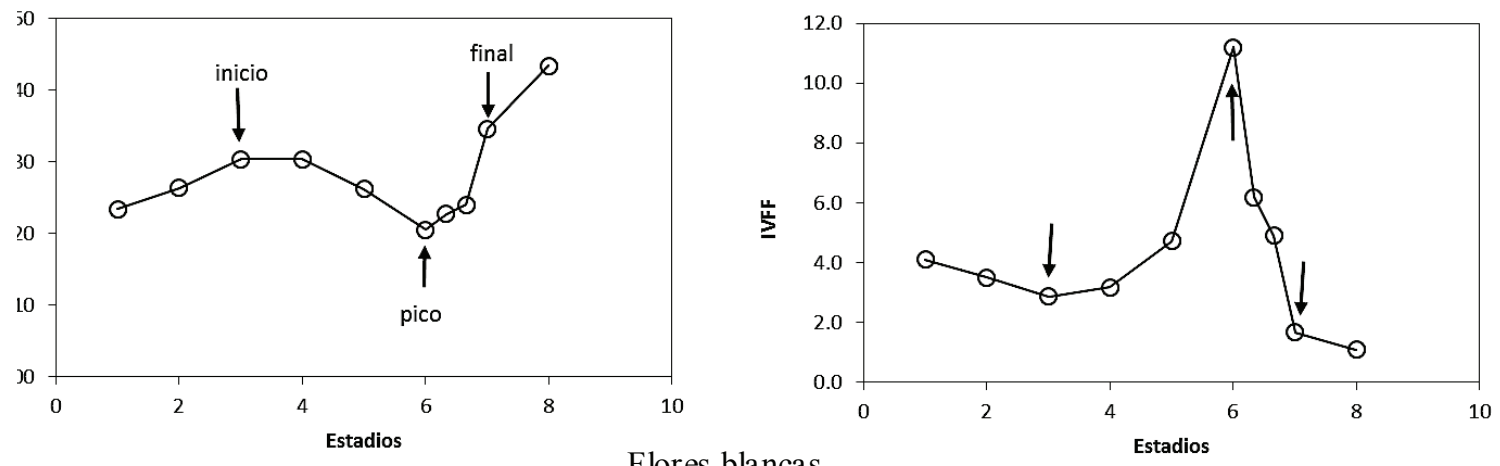

Flores blancas
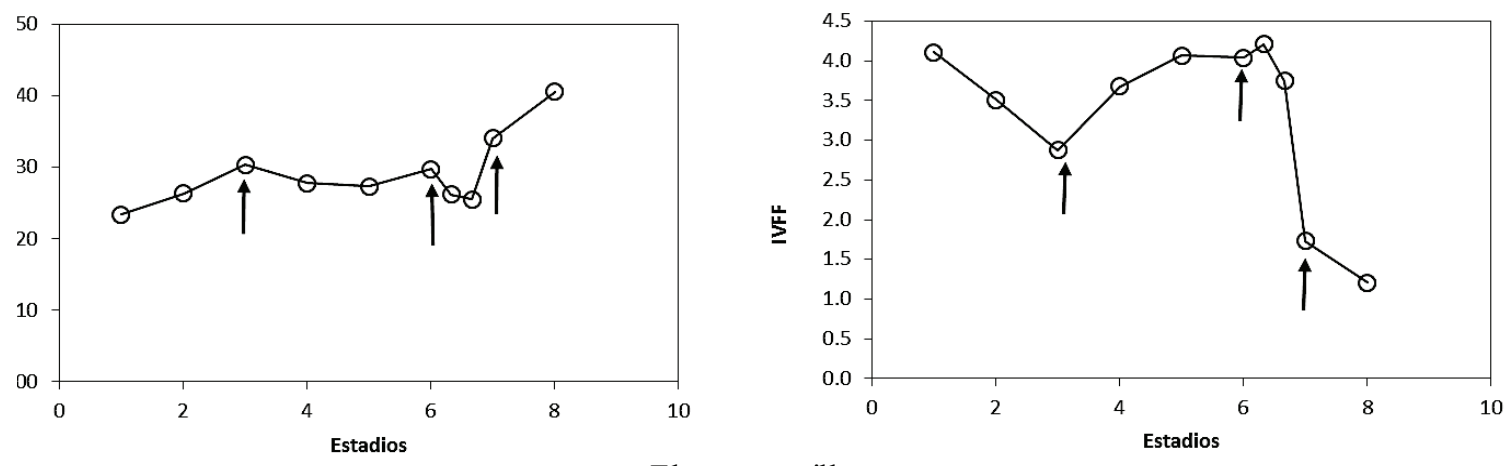

Flores amarillas

Figura 5. Patrones del IVISn e IVFF para diferentes estadios fenológicos, en el que las flechas indican el inicio, pico y final de la floración (adaptado de Casiano y Paz, 2014).

Para discriminar la intensidad de la floración, con relación a diferentes niveles de follaje, es necesario usar un índice de vegetación espectral que se relacione con la cobertura floral, tal como el propuesto por Chen et al. (2009), pero, desafortunadamente, este índice requiere de bandas espectrales angostas y no disponibles en el sensor MODIS y AVHRR a la resolución espacial

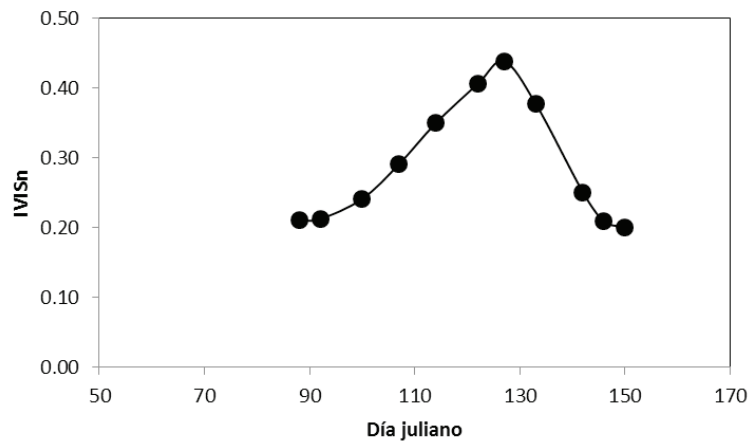

de interés ( $250 \mathrm{~m}$ y $1100 \mathrm{~m}$, a nadir), de tal manera que reviste importancia establecer una relación con el índice IVFF, por eso este trabajo revisó un modelo fenológico floración-follaje de desarrollo previo y se presenta un nuevo modelo que incluye el uso de un índice de vegetación de floración y follaje (IVFF) para caracterizar y diferenciar los patrones fenológicos.

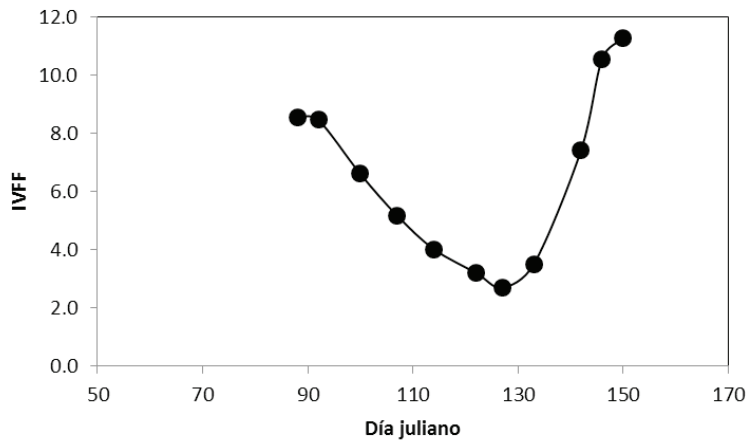

Figura 6. Evolución temporal de los patrones del IVISn e IVFF para el crecimiento del follaje y su senescencia en un cultivo de frijol (adaptado de Reyes et al., 2011). 


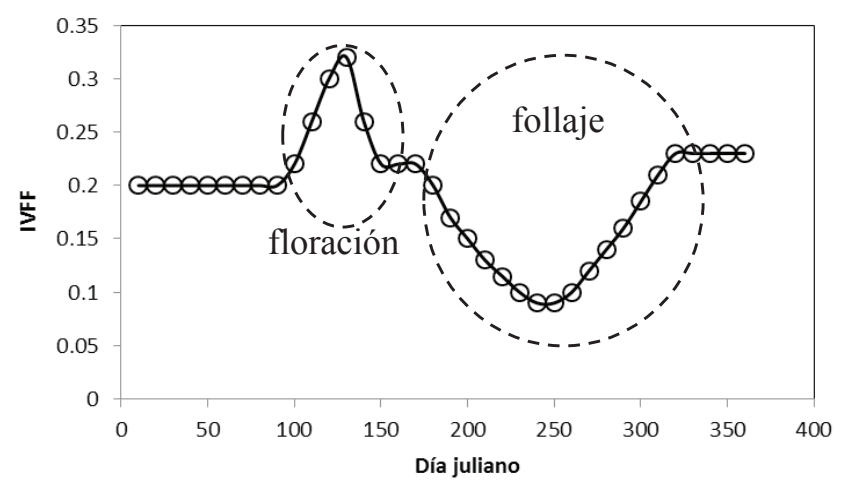

Figura 7. Estadios de la floración y follaje en un bosque tropical caducifolio.

\section{MATERIALES Y MÉTODOS}

El análisis de las reflectancias e índices de vegetación, con relación a diferentes niveles de floración, puede hacerse a partir de experimentos con diferentes componentes de las plantas, tales como tallos, hojas, yemas, brotes y flores (Ge et al., 2006; Wouters et al., 2013; Casiano y Paz, 2014), o bien, a través de la observación directa de la floración (Hunt y Williams, 2006; Chen et al., 2009). En el experimento que se realizó para el presente trabajo, se utilizó un enfoque híbrido, compuesto por matorrales de bajo porte, solo con follaje y, follaje con adición artificial de flores blancas, dado que el patrón espectral es similar al de las flores amarillas y lilas (Casiano y Paz, 2014).
El experimento tuvo lugar en San Luis Potosí en junio de 2014 en el paraje La presa de San José, cuyas coordenadas son $22^{\circ} 08^{\prime} 55.27^{\prime \prime} \mathrm{N}$ y $-101^{\circ} 01^{\prime}$ 53.63" O. Se utilizaron arbustos de la planta conocida coloquialmente como "rodadora" (Salsola collina), Figuras $8 \mathrm{a}$ y $8 \mathrm{~b}$, así como flores blancas de crisantemo y margarita (Chrysanthemum parthenium var. Satimex y Chrysanthemun leaucanthemum, respectivamente), Figuras $8 \mathrm{c}$ y $8 \mathrm{~d}$, mismas que se colocaron sobre el follaje del arbusto (Figura 8e).

Las mediciones de reflectancia espectral y cobertura floral (CF) se llevaron a cabo bajo condiciones de cielo soleado, con ángulos cenitales solares entre 1 y 30 grados (junio de 2014), con un radiómetro multiespectral portátil CROPSCANMR, modelo MSR5-590, de fabricación estadounidense, el cual mide reflectancias desde el dosel en cinco regiones del espectro electromagnético $(485,560,660,830,1650$ $\mathrm{nm}) \mathrm{y}$, cuyo campo de visión del sensor es de $28^{\circ}$. El radiómetro se instaló en un bastón telescópico en posición cenital a $3 \mathrm{~m}$ sobre el suelo, para cubrir un área de $1.76 \mathrm{~m}^{2}$ (área efectiva de medición, AEM). La reflectancia final, para cada punto de muestreo, fue el promedio de tres repeticiones.

Para cada patrón artificial de floración (PAF) del experimento, se pusieron flores blancas de crisantemo y margarita sobre plantas jóvenes, de un metro de altura, de rodadora (Salsola collina). Cada PAF se estableció en una parcela de $2 \times 2 \mathrm{~m}$, en porcentajes de cobertura de rodadora (representación nominal de

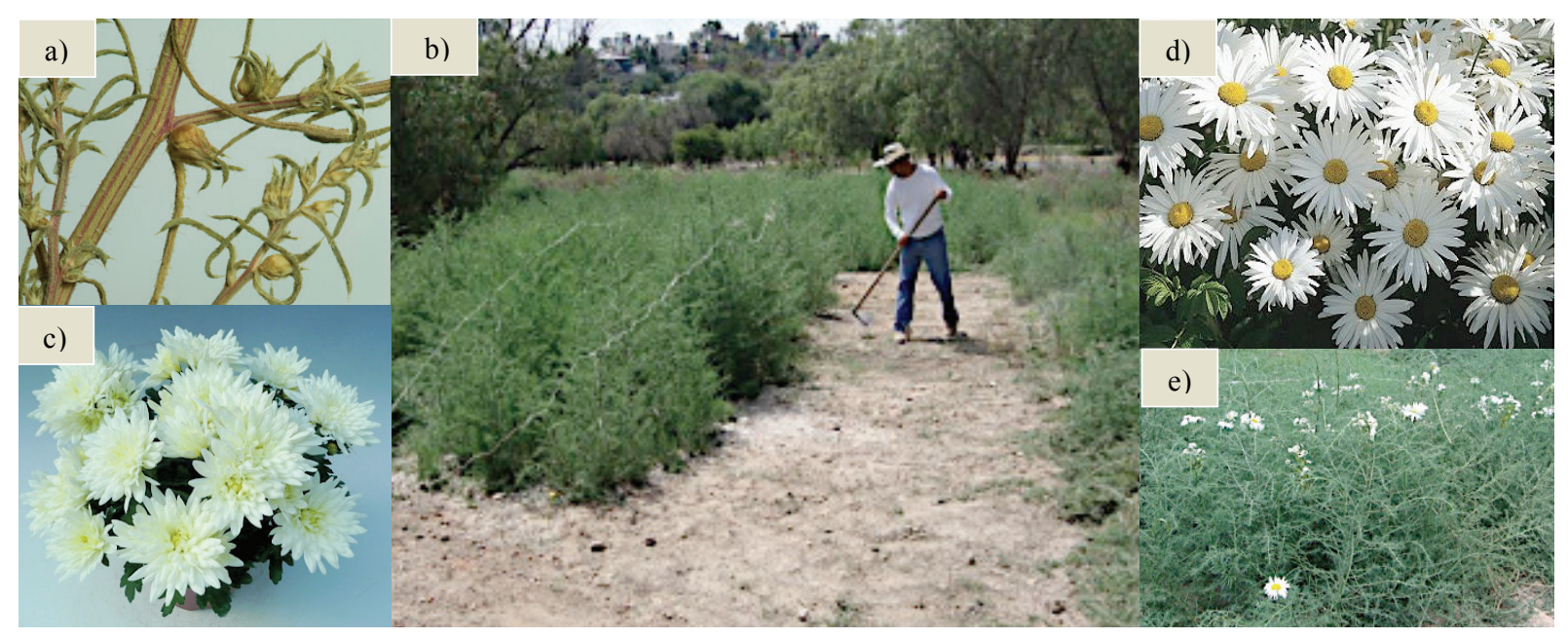

Figura 8. (a y b) Salsola collina o "rodadora"; (c) flores blancas de crisantemo (Chrysanthemum parthenium var. Satimex); (d) flores blancas de margarita (Chrysanthemun leaucanthemum); (e) establecimiento del experimento. 
la vegetación subyacente) de 0 (solo suelo), 60 y 80 . La colocación de flores sobre la rodadora, para cada PAF, incluyó únicamente porcentajes bajos $(<15 \%)$ del área de medición del radiómetro, con la finalidad de representar la situación de follaje con baja cobertura de flores. Además de la variación en el porcentaje de flores, se incluyeron dos tipos de distribución espacial: aleatoria y en grupos. El diseño de escenarios de cada PAF se muestra en la Figura 9.

La CF para cada PAF se estimó mediante técnicas estadísticas de clasificación supervisada, a partir de las fotografías digitales a nadir, una vez que se eliminó la sombra del radiómetro sobre el área de medición. Las coberturas obtenidas por las técnicas de clasificación supervisada fueron revisadas hasta lograr resultados aceptables.

\section{RESULTADOS Y DISCUSIÓN}

\section{Resultados}

La relación entre la cobertura del follaje y el IVFF (Figura 10), mostró una relación inversa a la ley de Beer-Lambert, totalmente acorde con la Figura 7, para el caso de un IV de solo follaje. La regresión estadística se forzó a pasar por el punto $(0,100)$ (Figura 10); lo que implica que cuando el IVFF tiene un valor de 0 , la cobertura de la vegetación es del $100 \%$.

La relación entre la CF y el IVFF es lineal y depende de la cobertura del follaje (Figura 3), lo que confirma que el IVFF es una sólida opción para estimar la CF en el follaje, particularmente para el caso de pocas flores, que es muy difícil de detectar por otros métodos.

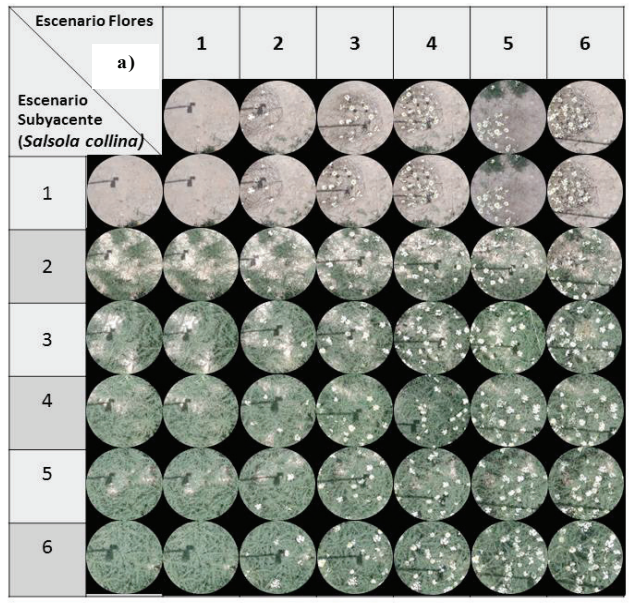

Aunado a lo anterior, el que el IVFF muestre poca variación cuando se usa una cobertura de follaje fija (debido a la transformación logarítmica que se usó en Rn e IRCn), implica que el IVFF puede transformarse a escala normal para amplificar las variaciones (Figura 11).

Cuando la CF presenta un arreglo espacial en grupos (Figura 12), la tendencia es similar a la del arreglo aleatorio. La posición de las flores modifica los parámetros de las relaciones, pero se conserva el patrón lineal de correlación.

Una forma de eliminar la constante aditiva de la relación lineal entre el IVFF y la $\mathrm{CF}$ es recentrar el IVFF (quitar el valor asociado al follaje, $C F=0$ ). En la Figura 13 se muestran los resultados de aplicar dicho esquema al caso de las flores con disposición aleatoria $\mathrm{y}$, en donde la cantidad de follaje corresponde a la pendiente (regresiones forzadas a pasar por el origen). Las pendientes de las relaciones de la Figura 13 están relacionadas linealmente con la cobertura del follaje.

Para generar una relación razonablemente general que permita estimar la CF con relación al IVFF recentrado, la CF del follaje se calcula como el porcentaje del área cubierta por las flores en relación al área del follaje y no del área de medición efectiva. La Figura 14 muestra el uso de la CF del follaje en relación con el IVFF recentrado.

Todos los resultados que se obtuvieron de la fase de validación experimental demuestran que el IVFF puede usarse para estimar la cantidad de área de floración en relación al área efectiva de medición (pixeles en imágenes de satélite) y el área del follaje. En el primer caso, la relación entre el IVFF (después de recentrarlo) depende de la cantidad de follaje en el área efectiva de

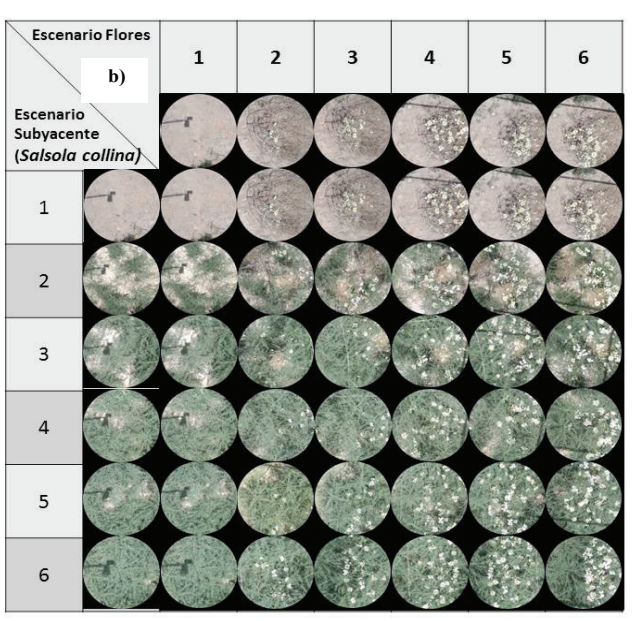

Figura 9. Conjunto fotográfico de AEM, para los dos tipos de distribución espacial de las flores, aleatorio y en grupos. 


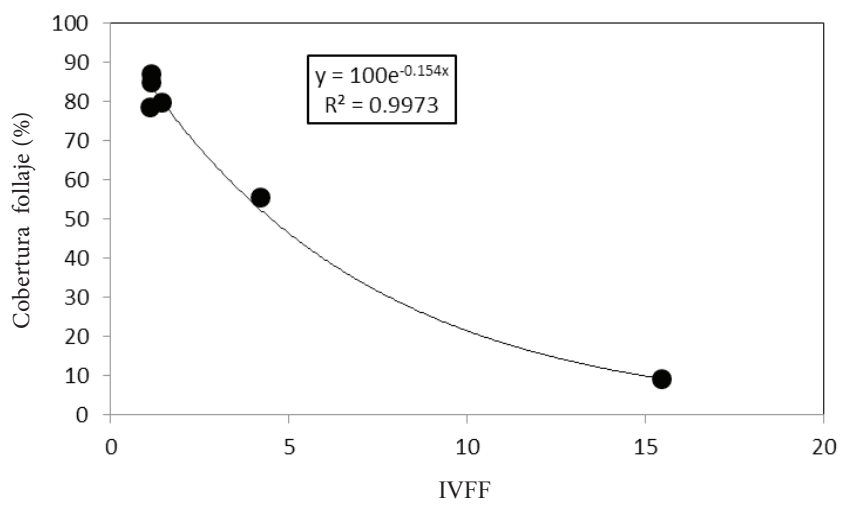

Figura 10. Relación entre el IVFF y la cobertura del follaje en los experimentos de rodadora con diferentes coberturas de flores blancas.

medición, lo que no ocurre en el segundo, aún cuando se requiere estimar la cobertura del follaje en el área o pixel, lo cual se puede realizar fácilmente con sensores remotos que midan esta cobertura antes del inicio de la floración.

Tanto el desarrollo teórico como la evidencia experimental, demuestran que la aplicación de la tecnología delos sensores remotos paralacaracterización de los patrones fenológicos de la floración y el follaje, es eficaz, además de que permite asociar las observaciones con estimaciones de la cobertura foliar. Una ventaja más es que, a diferencia del índice de floración introducido por Chen et al. (2009), el IVFF es únicamente función de las bandas del rojo e infrarrojo cercano, mismas que se encuentran disponibles en la gran mayoría de los sensores en plataformas espaciales, lo que hace asequible su utilización.

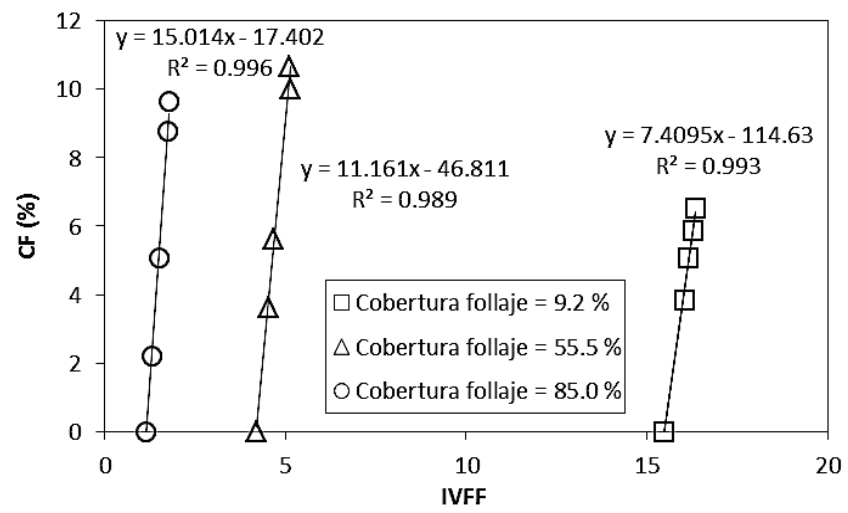

Figura 11. Relación entre el IVFF y la cobertura floral (CF) para tres coberturas del follaje con diferentes $C F$ y arreglo espacial de las flores aleatorio.

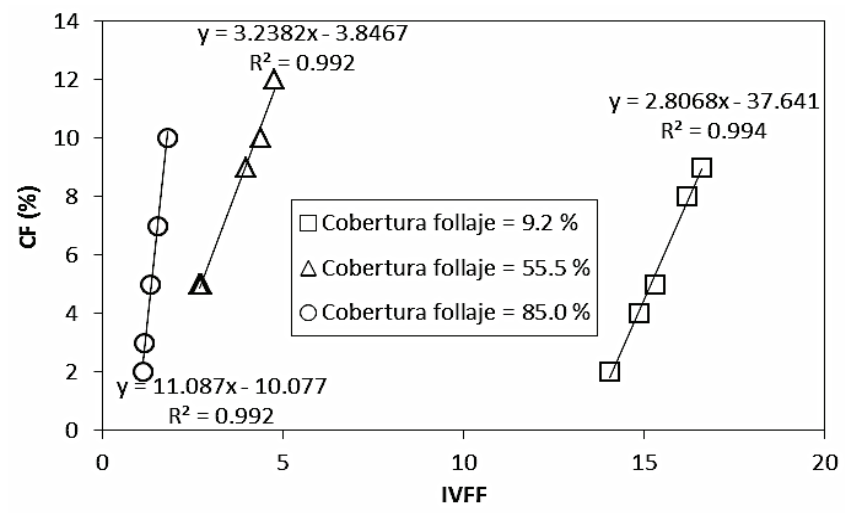

Figura 12. Relación entre el IVFF y la cobertura floral (CF) para tres coberturas del follaje con diferentes CF y arreglo espacial de las flores en grupo.

\section{Discusión}

\section{Sincronización de la Floración y el Follaje}

En los estudios satelitales regionales y globales de caracterización de la fenología de la vegetación, para bosques y otros tipos de vegetación, a diferencia de lo que se muestra en la Figura 3, solo se detecta el patrón de la Figura 1b (Shabanov et al., 2002; Stöckli y Vidale, 2004; Zhang et al., 2004; Fisher et al., 2006; Zhang et al., 2006), con excepción de los trabajos de Huete et al. (2006) y Saleska et al. (2007) quienes observaron crecimiento del follaje, durante el periodo seco, en bosques tropicales de la Amazonia. Samanta et al. (2010) critican este resultado y lo exponen como un artefacto de los efectos atmosféricos de los aerosoles; pero, posteriormente, Huete y Saleska (2010) generaron

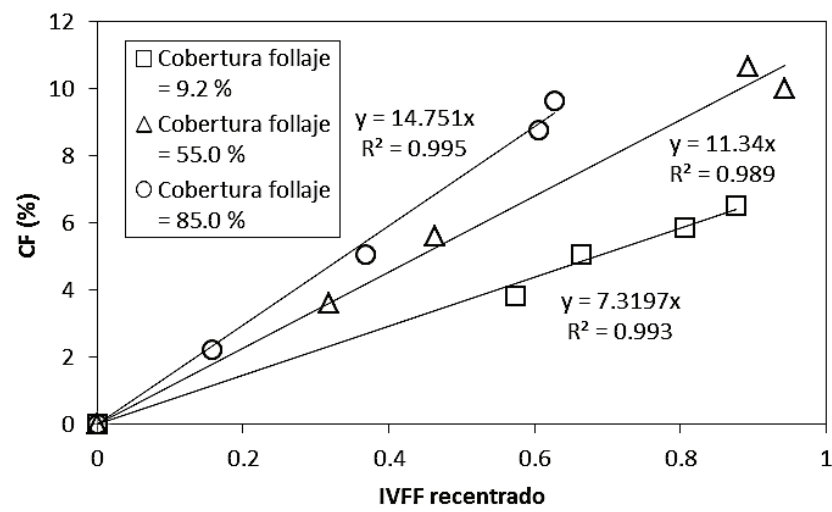

Figura 13. Relación entre el IVFF recentrado y la cobertura floral (CF) para tres coberturas del follaje con diferentes niveles de CF y arreglo espacial de las flores aleatorio. 


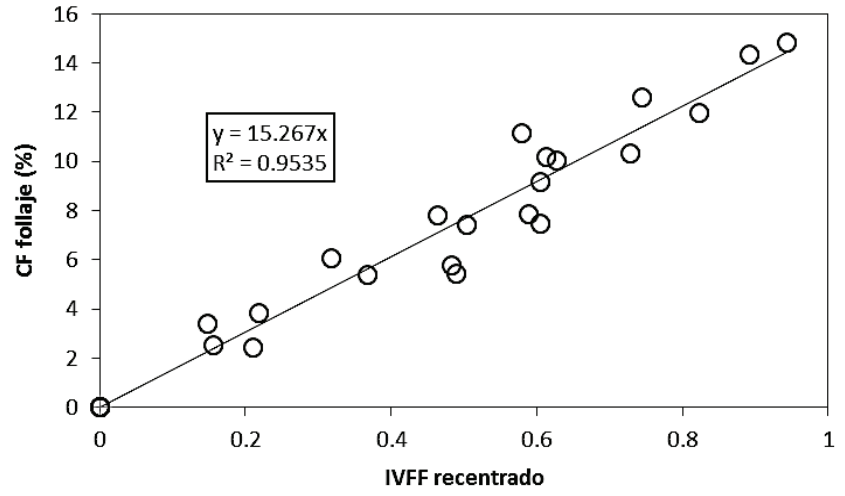

Figura 14. Relación entre el IVFF recentrado y la cobertura floral (CF) del follaje para todos los datos del experimento.

evidencia de la robustez del patrón al procesar datos espectrales de mejor calidad y sin efectos atmosféricos.

En los árboles, de acuerdo con Hallé et al. (1978), la transición del meristemo vegetativo a la iniciación de la floración, tiene dos vías: pasar del meristemo vegetativo al eje de floración $\mathrm{y}$, continuar el meristemo vegetativo con floración lateral; motivo por el cual, la producción de hojas y flores puede ser simultánea, ya que los mismos meristemos que producen yemas foliares, también producen flores (Borchert, 1983).

La sincronización de la aparición de flores y hojas, durante el periodo seco, puede darse con superposición (Bullock y Solis-Magallanes, 1990; Kikim y Yadava, 2001; Marques et al., 2004) o tener un desfase temporal (Lobo et al., 2003; Valdez-Hernández et al., 2010; Liuth et al., 2013). La Figura 15 muestra en forma esquemática ambos patrones.

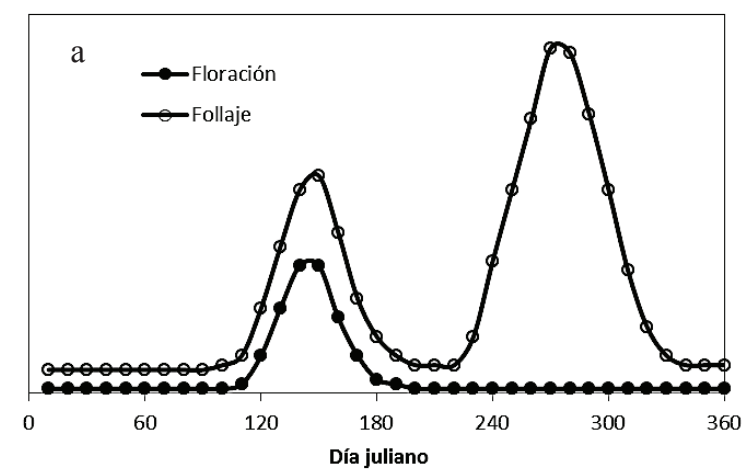

El modelo que se presenta en la Figura 15 considera únicamente eventos de floración detectables (masivos), por lo que aún cuando los eventos de floración de las especies pueden darse por detonaciones parciales, el patrón de agregación es continuo (van Schaik et al., 1993). Durante la época seca se induce la floración, generalmente, como resultado de eventos de precipitación y por el nivel de estrés hídrico (Opler et al., 1976; Heidemann 1989; Reich, 1995; Borchert, 1996). En la mayoría de los casos existe una sincronización de la floración entre especies (Opler et al., 1976; Rathcke y Lacey, 1985; Boyle y Bronstein, 2012), lo que sugiere una restricción filogenética (Kochner y Handel, 1986; Bawa et al., 2003; Chuine, 2010).

El pico del follaje (y floración), en época seca, suele coincidir con el pico de irradianza solar (van Schaik et al., 1993; Huete et al., 2006), lo que sustenta que se trata de una estrategia óptima para la vegetación, ya que incrementa la eficiencia fotosintética y reduce los costos de transferencia de los asimilados entre los órganos de las plantas (Wright y van Schaik, 1994; Kikuzawa, 1995). Tanto en bosques (Nilson et al., 2012) como en herbáceas (Hunt y Williams, 2006), se observa un desfase temporal entre los picos de floración y follaje (Figura 8b), que concierne a los patrones entre las bandas del Rn e IRCn, hecho que denota un patrón similar al de la Figura 3.

El índice de vegetación del follaje y floración introducido en este trabajo tiene la ventaja de poder detectar patrones de crecimiento sincronizado, o no, de follaje y vegetación, dado que puede medir valores bajos de cubrimiento de floración en el follaje, permitiendo así el monitoreo de los eventos de floración, masivos o no.

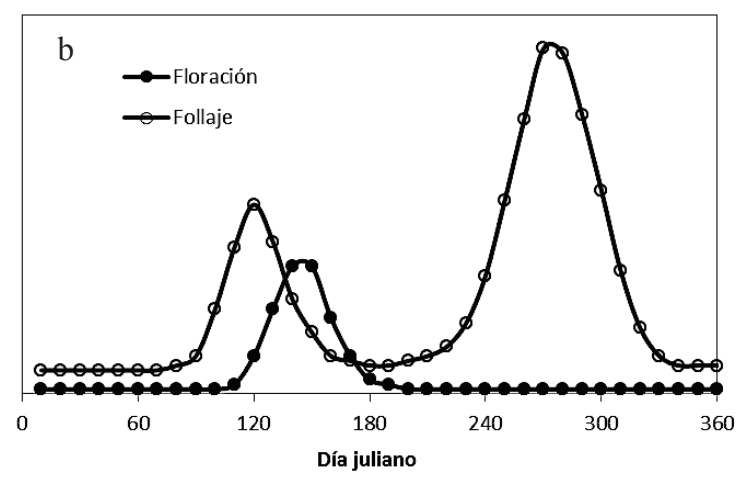

Figura 15. Patrones de sincronización de la floración y el follaje: a) con superposición en tiempo y b) con desfase temporal. 


\section{CONCLUSIONES}

- Se desarrolló un modelo fenológico para floración y follaje, que incluye los patrones temporales asociados a dichos eventos, así como un índice de vegetación de floración y follaje (IVFF) capaz de diferenciar los patrones espectrales de las bandas del rojo e infrarrojo cercano. El desarrollo propuesto permitió establecer correlaciones claras entre el IVFF y la cobertura floral de la vegetación con diferentes coberturas aéreas, por lo que es eficaz aún cuando el porcentaje de floración es bajo y no puede detectarse por otras metodologías. El que sólo se utilicen las bandas del rojo y el infrarrojo cercano, lo convierte en un método altamente asequible para la mayoría de las aplicaciones de sensores remotos. - En trabajos posteriores de los autores se presentará la aplicación de la metodología desarrollada para la caracterización de la fenología de la vegetación en México usando el sensor MODIS.

\section{LITERATURA CITADA}

Atkinson, P. M., C. Jeganathan, J. Dash, and C. Atzberger. 2012. Inter-comparison of four models for smoothing satellite sensor time-series data to estimate vegetation phenology. Remote Sens Environ. 123: 400-417. doi: 10.1016/j.rse.2012.04.001.

Bawa, K. S. 1990. Plant-pollinator interactions in tropical rain forests. Annu. Rev. Ecol. Syst. 21: 399-422. doi: 10.1146/ annurev.es.21.110190.002151.

Bawa, K. S., H. Kang, and M. H. Grayum. 2003. Relationships among time, frequency, and duration of flowering in tropical rain forest trees. Am. J. Bot. 90: 877-887. doi: 10.3732/ ajb.90.6.877.

Bolaños G., M. A. y F. Paz P. 2010. Modelación general de los efectos de la geometría de iluminación-visión en la reflectancia de pastizales. Rev. Mex. Cienc. Pecu. 1: 349-361.

Borchert, R. 1983. Phenology and control of flowering in tropical trees. Biotropica 15: 81-89. doi: 10.2307/2387949.

Borchert, R. 1996. Phenology and flowering periodicity of Neotropical dry forest species: Evidence from herbarium collections. J. Trop. Ecol. 12: 65-80. doi: 10.1017/ S0266467400009317.

Boyle, W. A. and J. L. Bronstein. 2012. Phenology of tropical understory trees: patterns and correlates. Rev. Biol. Trop. (Int. J. Trop. Biol. ISSN-0034-7444) 60: 1415-1430.

Bullock, S. H. and J. A. Solis-Magallanes. 1990. Phenology of canopy trees of a tropical deciduous forest in Mexico. Biotropica 22: 22-35. doi: 10.2307/2388716.

Casiano, M. y F. Paz. 2014. Sobre la caracterización fenológica de bosques tropicales usando información espectral: experimentos con componentes. Terra Latinoamericana 32: 259-271.

Chen, J., M. Shen, X. Zhu, and Y. Tang. 2009. Indicator of flower status derived from in situ hyperspectral measurement in an alpine meadow on the Tibetan Plateau. Ecol. Indicat. 9: 818823. doi: 10.1016/j.ecolind.2008.09.009.
Chuine, I. 2010. Why does phenology drive species distribution? Phil. Trans. R. Soc. B 365: 3149-3160. doi: 10.1098/ rstb.2010.0142.

Cleland, E. E., I. Chuine, A. Menzel, H. A. Mooney, and M. D. Schwartz. 2007. Shifting plant phenology in response to global change. Trends Ecol. Evol. 22: 357-365. doi: 10.1016/j. tree.2007.04.003.

Cuba, N., J. Rogan, Z. Christman, C. A. Williams, L. C. Schneider, D. Lawrence, and M. Millones. 2013. Modeling dry season deciduousness in Mexican Yucatán forest using MODIS EVI data (2000-2011). GISci. Remote Sens. 50: 26-49. doi: 10.1080/15481603.2013.778559.

Fisher, J. I., J. F. Mustard, and M. V. Vadeboncoeur. 2006. Green leaf phenology at Landsat resolution: Scaling from the field to the satellite. Remote Sens. Environ. 100: 265-279. doi: 10.1016/j.rse.2005.10.022.

Ge, S., J. Everitt, R. Carruthers, P. Gong, and G. Anderson. 2006. Hyperspectral characteristics of canopy components and structure for phonological assessment of an invasive weed. Environ. Monitor. Assess. 120:109-126. doi: 10.1007/s10661005-9052-1.

Geerken, R. A. 2009. An algorithm to classify and monitor seasonal variations in vegetation phenologies and their inter-annual change. ISPRS J. Photogram. Remote Sens. 64: 422-431. 10.1016/j.isprsjprs.2009.03.001.

Gentry, A. H. 1974. Flowering phenology and diversity in tropical Bignoniaceae. Biotropica 6: 64-68. doi: 10.2307/2989698.

Hallé, F., R. A. A. Oldeman, and P. B. Tomlinson. 1978. Tropical trees and forests: An architectural analysis. Springer-Verlag. New York, NY, USA.

Heideman, P. D. 1989. Temporal and spatial variation in the phenology of flowering and fruiting in a tropical rainforest. J. Ecol. 77: 1059-1079. doi: 10.2307/2260823.

Huete, A. R., K. Didan, Y. E. Shimabukuro, P. Ratana, S. R. Saleska, L. R. Hutyra, W. Yang, R. R. Nemani, and R. Myneni. 2006. Amazon rainforests green-up with sunlight in dry season. Geophys. Res. Lett. 33: L06405. doi: 10.1029/2005GL025583.

Huete, A. R. and S. R. Saleska. 2010. Remote sensing of tropical forest phenology: Issues and controversies. Int. Arch. Photogram. Remote Sens. Spat. Informat. Sci. 38: 539-542.

Hunt, E. R. and A. E. P. Williams. 2006. Detection of flowering leafy spurge with satellite multispectral imagery. Rangel. Ecol. Manage. 59: 494-499. doi: 10.2111/05-216R.1.

Jiang, Z., A. R. Huete, K. Didan, and T. Miura. 2008. Development of a two-band enhanced vegetation index without a blue band. Remote Sens. Environ. 112: 3833-3845. doi: 10.1016/j. rse.2008.06.006.

Kathuroju, N., M. A. White, J. Symanzik, M. D. Schwartz, J. A. Powell, and R. R. Nemani. 2007. On the use of the advanced very high resolution radiometer for development of prognostic land surface phenology models. Ecol. Modell. 201: 144-156. doi: 10.1016/j.ecolmodel.2006.09.011.

Kaufman, Y. J. and D. Tanre. 1996. Strategy for direct and indirect methods for correcting the aerosol effect on remote sensing: From AVHRR to EOS-MODIS. Remote Sens. Environ. 55: 65-79. doi: 10.1016/0034-4257(95)00193-X.

Kikuzawa, C. P. 1995. Leaf phenology as an optimal strategy for carbon gains in plants. Can. J. Bot. 73: 158-163. doi: 10.1139/ b95-019. 
Kikim, A. and P. S. Yadava. 2001. Phenology of tree species in subtropical forests of Manipur in north eastern India. Int. Soc. Trop. Ecol. 42: 269-276.

Kochner, J. P. and S. N. Handel. 1986. Constraints and competition in the evolution of flowering phenology. Ecol. Monogr. 56: 303-325. doi: 10.2307/1942549.

Liu, H. Q. and A. Huete. 1995. A feedback based modification of the NDVI to minimize canopy background and atmospheric noise. IEEE Trans. Geosci. Remote Sens. 33: 457-465. doi: $10.1109 / 36.377946$.

Liuth, H. S., D. C. Talora, and A. M. Amorim. 2013. Phenological synchrony and seasonality of understory Rubiaceae in the Atlantic Forest, Bahia, Brazil. Acta Bot. Bras. 27: 195-204. doi: 10.1590/S0102-33062013000100019.

Lobo, J. A., M. Quesada, K. E. Stoner, E. J. Fuchs, Y. HerreriasDiego, J. Rojas, and G. Saborio. 2003. Factors affecting phenological patterns in bombacaceous trees in seasonal forests in Costa Rica and Mexico. Am. J. Bot. 90: 1054-1063. doi: 10.3732/ajb.90.7.1054.

Marques, M. C. M., J. J. Roper, and A. P. B. Salvalaggio. 2004. Phenological patterns among plant life-forms in a subtropical forest in southern Brazil. Plant Ecol. 173: 203-213. doi: 10.1023/B:VEGE.0000029325.85031.90.

Newstrom, L. E., G. W. Frankie, and H. G. Baker. 1994. A new classification for plant phenology based on flowering patterns in lowland tropical rain forests trees at La Selva, Costa Rica. Biotropica 26: 141-159. doi: 10.2307/2388804.

Nilson, T., M. Rautiainen, J. Pisek, and U. Peterson. 2012. Seasonal reflectance courses of forests. New Adv. Contribut. Forest. Res. Chapter 3: 33-58. doi: 10.57727/34962.

Opler, P. A., G. W. Frankie, and H. G. Baker. 1976. Rainfall as a factor in the release, timing and synchronization of anthesis by tropical trees and shrubs. J. Biogeogr. 3: 231-236. doi: $10.2307 / 3038013$

Opler, P. A., G. W. Frankie, and H. G. Barker. 1980. Comparative phonological studies of treelet and shrub species in tropical wet and dry forests in the lowland of Costa Rica. J. Ecol. 68: 167-188.

Parihar, J. S., S. Goroshi, R. Singh, N. S. R. Krishnayya, M. B. Sirsayya, A. Kumar, L. S. Rawat, and A. Sonakia. 2013. Observation of forest phenology using field-based digital photography and satellite data. Current Sci. 105: 1740-1747.

Paz, F., M. Reyes y E. Medrano. 2011. Diseño de índices espectrales de la vegetación usando curvas iso-suelo. Agrociencia 45: 121-134.

Peñuelas, J. and I. Fillela. 2001. Phenology-responses to a warming world. Science 294: 793-795. doi: 10.1126/science.1066860.

Rathcke, B. and E. Lacey.1985. Phenological patterns of terrestrial plants. Ann. Rev. Ecol. Syst. 16: 179-214. doi: 10.1146/ annurev.es.16.110185.001143.

Reich, P. B. 1995. Phenology of tropical forest: patterns, causes and consequences. Can. J. Bot. 73: 164-174. doi: 0.1139/b95-020.

Reyes, M., F. Paz, M. Casiano, F. Pascual, M. I. Marín y E. Rubiños. 2011. Caracterización del efecto de estrés usando índices espectrales de la vegetación para la estimación de variables relacionadas con la biomasa aérea. Agrociencia 45: 221-233.

Richardson, A. D., B. H. Braswell, D. Y. Hollinger, J. P. Jenkins, and S. V. Ollinger.2009. Near-surface remote sensing of spatial and temporal variation in canopy phenology. Ecol. Applic. 19: 1417-1428. doi: 10.1890/08-2022.1.
Rouse, J. W., R. H. Haas, J. A. Schell, D. W. Deering, and J. C. Harlan. 1974. Monitoring the vernal advancement of retrogradation of natural vegetation. Final Report, E75-10354, NASA-CR-144661, RSC-1978-4. NASA. Washington, DC, USA.

Running, S. W. and R. R. Nemani. 1991. Regional hydrologic and carbon balance response of forest resulting from potential climate change. Clim. Change 19: 349-368. doi: 10.1007/ BF00151173.

Saleska, S. R., K. Didan, A. R. Huete, and H. R. da Rocha. 2007. Amazon forests green up during 2005 drought. Science 318: 612. doi:10.1126/science. 1146663.

Sakai, S. 2001. Phenological diversity in tropical forests. Popul. Ecol. 43: 77-86. doi: doi.org/10.1007/PL00012018.

Samanta, A., S. Ganguly, H. Hashimoto, S. Devadiga, E. Vermote, Y. Knyazikhin, R. R. Nemani, and R. B. Myneni. 2010. Amazon forests did not Green-up during 2005 droughts. Geophys. Res. Lett. doi: 10.1029/2009GL042154.

Schwartz, M. D., R. Ahas, and A. Aasa. 2006. Onset of spring starting earlier across the Northern Hemisphere. Global Change Biol. 12: 343-351. doi: 10.1111/j.1365-2486.2005.01097.x.

Shabanov, N. V., L. Zhou, Y. Knyazikhin, R. B. Myneni, and C. J. Tucker. 2002. Analysis of interannual changes in northern vegetation activity observed in AVHRR data from 1981 to 1994. IEEE Trans. Geosci. Remote Sens. 40: 115-130. doi: $10.1109 / 36.981354$.

Stöckli, and P. L. Vidale. 2004. European plant phenology and climate as seen in a 20-year AVHRR land-surface parameter dataset. Int. J. Remote Sens. 25: 3303-3330. doi: 10.1080/01431160310001618149.

Tucker, C. J. 1979. Red and photographic infrared linear combination for monitoring vegetation. Remote Sens. Environ. 8: 127-150. doi: 10.1016/0034-4257(79)90013-0.

Valdez-Hernández, M., J. L. Andrade, P. C. Jackson, and M. Rebolledo-Vieyra. 2010. Phenology of five tree species of a tropical dry forest in Yucatan, Mexico: Effects of environmental and physiological factors. Plant Soil 329: 155171. doi: 10.1007/s11104-009-0142-7.

Van Schaik, C. P., J. W. Terborgh, and S. J. Wright. 1993. The phenology of tropical forests: Adaptive significance and consequences for primary consumers. Annu. Rev. Ecol. Syst. 24: 353-377. doi: 10.1146/annurev.es.24.110193.002033.

White, M. A., S. W. Running, and P. E. Thornton. 1999. The impact of growing-season length variability on carbon assimilation and evapotranspiration over 88 years in the eastern US deciduous forest. Int. J. Biometeorol. 42: 139-145. doi: $10.1007 / \mathrm{s} 004840050097$.

Wouters, N., B. de Ketelaere, J. de Baerdemaeker, and W. Saeys. 2013. Hyperspectral waveband selection for automatic detection of floral pear buds. Precision Agric. 14: 86-98. doi: doi.org/10.1007/s11119-012-9279-0.

Wright, S. J. and C. P. van Schaik. 1994. Light and the phenology of tropical trees. The Am. Natural. 143: 192-199.

Zhang, P., B. Anderson, M. Barlow, B. Tan, and R. B. Myneni. 2004. Climate-related vegetation characteristics derived from moderate resolution imaging spectroradiometer (MODIS) leaf area index and normalized difference vegetation index. J. Geophys. Res. 109, D20105. doi:10.1029/2004JD004720. 
Zhang, X., M. A. Friedl, and C. B. Schaaf. 2006. Global vegetation phenology from moderate resolution imaging spectroradiometer (MODIS): evaluation of global patterns and comparison with in situ measurements. J. Geophys. Res. 111, G04017. doi:10.1029/2006J6000217.

Zhang, X., M. A. Friedl, C. B. Schaaf, A. H. Strahler, J. C. F. Hodges, F. Gao, B. C. Reed, and A. Huete. 2003. Monitoring vegetation phenology using MODIS. Remote Sens. Environ. 84: 471-475. doi: 10.1016/S0034-4257(02)00135-9.

Zhang, X., M. A. Friedl, B. Tan, M. D. Goldberg, and Y. Yu. 2012. Long-term detection of global vegetation phenology from satellite instruments. pp. 297-320. In: X. Zhang (ed.). Chapter 16, Phenology and climate change. ISBN 978-953-51-0336-3. In tech open access publisher. doi:10.5772/39197. 